\title{
Le Droit Suisse des Sociétés en 2018: Modifications législatives, jurisprudence et doctrine
}

\author{
Damiano Canapa / Arthur Grisoni / Line Elodie Derungs *
}

Cette contribution propose un aperçu, pour l'année 2018, des principales modifications législatives, de la jurisprudence du Tribunal fédéral [TF] et des ouvrages de doctrine publiés en droit suisse des sociétés. La section consacrée aux modifications législatives (I.) présente principalement les derniers développements de la modification projetée du droit de la société anonyme [SA]. Les résumés de jurisprudence font l'objet de la section suivante (II.). Finalement, les ouvrages de doctrine (III.) sont recensés par thème.

I. Modifications législatives 301

1. Loi fédérale sur les aides financières aux organisations de cautionnement en faveur des petites et moyennes entreprises 301

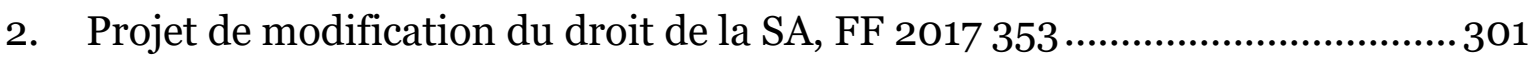

II. Jurisprudence 303

1. Sociéte simple 303

2. Société anonyme 304

3. Société à responsabilité limitée. 313

4. Société coopérative 315

III. Doctrine 315

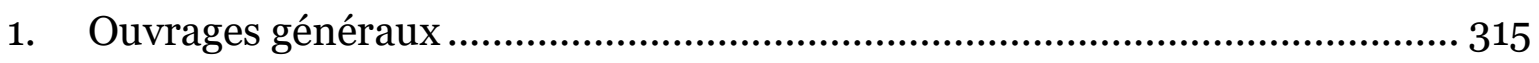

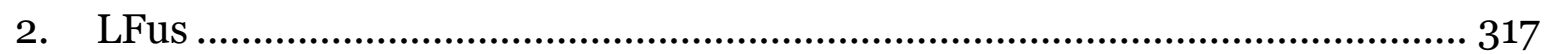

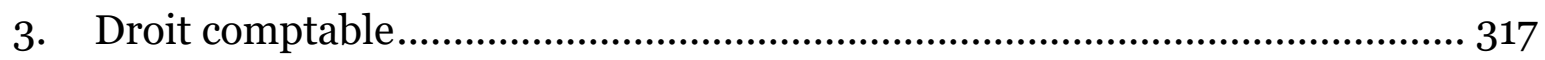

Citation: $\quad$ Damiano Canapa/Arthur Grisoni/Line Elodie Derungs, Le Droit Suisse des Sociétés en 2018: Modifications législatives, jurisprudence et doctrine, dans: sui-generis 2019, S. 300

URL: $\quad$ sui-generis.ch/111

DOI: $\quad$ https://doi.org/10.21257/sg.111

* Damiano Canapa (damiano.canapa@unil.ch), Professeur à l'Université de Lausanne, LL.M. (Bruges), LL.M. (Yale), Arthur Grisoni (arthur.grisoni@unil.ch), MLaw, Assistant doctorant à l'Université de Lausanne, Line Elodie Derungs (line.derungs@unil.ch), MLaw, Assistante doctorante à l'Université de Lausanne. Les auteurs remercient Monsieur Yvan Gisling, étudiant en droit, pour son aide dans la recherche, ainsi que Me Cédric Ballenegger pour sa relecture attentive de leur contribution.

Ce(tte) œuvre est mise à disposition selon les termes de la Licence Creative Commons Attribution Partage dans les Mêmes Conditions 4.o International. 


\section{Modifications législatives}

\section{Loi fédérale sur les aides finan- cières aux organisations de cau- tionnement en faveur des petites et moyennes entreprises ${ }^{1}$}

$1 \mathrm{Vu}$ le message du Conseil fédéral du 14 février $2018^{2}$, l'Assemblée fédérale a arrêté, le 14 décembre 2018, un projet modifiant la Loi fédérale du 6 octobre 2006 sur les aides financières aux organisations de cautionnement en faveur des petites et moyennes entreprises 3 . Cette modification concerne essentiellement trois points: «relèvement du plafond de cautionnement à 1 million de francs (art. 6); adaptation du principe de subsidiarité (art. 2) et réduction de la contribution de la Confédération aux frais administratifs dans le cadre de la répartition de l'excédent aux sociétaires (art. 7)»4.

1 Projet de modification de la Loi fédérale du 6 octobre 2006 sur les aides financières aux organisations de cautionnement en faveur des petites et moyennes entreprises (FF 2018 1281)

2 Message du 14 février 2018 sur la modification de la loi fédérale sur les aides financières aux organisations de cautionnement en faveur des petites et moyennes entreprises et sur l'abrogation de la loi fédérale sur l'octroi de cautionnements et de contributions au service de l'intérêt dans les régions de montagne et le milieu rural en général (FF 2018 1253).

3 Loi fédérale sur les aides financières aux organisations de cautionnement en faveur des PME du 6 octobre 2006 (RS 951.25).

4 Message du 14 février 2018 sur la modification de la loi fédérale sur les aides financières aux organisations de cautionnement en faveur des petites et moyennes entreprises et sur l'abrogation de la loi fédérale sur l'octroi de cautionnements et de contributions au service de l'intérêt dans les régions de montagne et le milieu rural en général (FF 2018 1255). Le délai référendaire a échu le 7 avril 2019 et la modification législative est entrée en vigueur 1 1er juillet 2019 (RO 2019 1781).

\section{Projet de modification du droit de la SA, FF $2017353^{5}$}

2 «Projet 1»: Pour rappel, le Conseil fédéral a publié, le 23 novembre 2016, son Message concernant la modification du code des obligations (Droit de la société anonyme) ${ }^{6}$. Ce projet a été adopté par le Conseil national le 15 juin 20187. A cette occasion, le Conseil national a également proposé un contre-projet indirect à l'initiative «Entreprises responsables pour protéger l'être humain et l'environnement». Le projet de modification du droit de la SA a alors été scindé en deux parties. La partie issue du Message du Conseil fédéral est devenue le «projet $1{ }^{8}$, tandis que les aspects en lien avec l'initiative «Entreprise responsables pour protéger l'être humain et l'environnement» ont été retirés du projet principal pour devenir le «projet 2»9 (voir ci-dessous).

3 En lien avec le «projet 1», le Conseil national a soutenu l'introduction de quotas de genres dans les directions (minimum 20\% de personnes du genre le moins représenté) et les conseils d'administration

5 Pour un état des lieux des points traités lors de cette révision, voir Cartier, Patricia, Droit du registre du commerce et domaines connexes: aperçu des travaux parlementaires et des nouveautés en 2017, REPRAX 2018, pp. 43-50; von der Crone, Hans Caspar/Grob, Thomas, Die virtuelle Generalversammlung, RSDA 2018, pp. 5-20; Sethe Rolf, Die Regelung von Interessenkonflikten im Aktienrecht de lege lata und de lege ferenda, RSDA 2018, pp. 375-392; Sethe, Rolf/Cetinkaya, Meltem, Sinn und Unsinn von Formerleichterungen im geplanten Aktienrecht, REPRAX 2018, pp. 153 ss.

6 Message du 23 novembre 2016 concernant la modification du code des obligations (FF 2017 353); à ce sujet, voir généralement: Damiano Canapa/Marleen Botterbrodt/Antoine Schneebeli, Le droit Suisse des Sociétés en 2017, (2018) sui generis, 297, 299, et les références citées.

7 BO CN 20181054 ss.

8 Proposition Bigler, cf. BO CN 20181068 ss, 1076.

9 Proposition Bigler, cf. BO CN 20181068 ss, 1076. 
(minimum 30\% de personnes du genre le moins représenté) des SA cotées en bourse. En relation avec la mise en œuvre de l'initiative contre les rémunérations abusives $^{10}$, le Conseil national a décidé de ne pas plafonner les bonus et de continuer d'autoriser certaines primes d'embauche. Des mesures visant à faciliter la fondation d'une SA et la modification de son capital ont également été adoptées, telle que la suppression de l'obligation d'authentifier l'acte constitutif de certaines $\mathrm{SA}$ «à structure simple» - soit les SA dont (1) le contenu des statuts se limite aux dispositions prévues à l'art. 626 al. $1 \mathrm{CO}$, (2) le capital-actions est fixé en francs et (3) les apports sont entièrement libérés en francs - et l'introduction du mécanisme de marge de fluctuation du capital. Ce dernier permettrait au conseil d'administration [CA] d'une SA, dans un délai de cinq ans, d'augmenter ou de réduire le capital-actions de la société dans certaines limites préalablement décidées par l'assemblée générale [AG].

4 Le 11 décembre 2018, le Conseil des Etats est entré en matière sur le «projet 1» et l'a renvoyé en commission. ${ }^{11}$

5 «Projet 2»: Le 18 mai 2018, la Commission des affaires juridiques du Conseil national a publié, dans le cadre de la révision du droit de la société anonyme, un rapport complémentaire sur les propositions de la commission en vue du dépôt d'un contre-projet indirect à l'initiative populaire «Entreprises responsables -

10 FF 2017353 (n. 6), pp. 400 ss.

11 Le Conseil des Etats a entre-temps adopté le «projet 1» le 19 juin 2019. Suite à cela, la Commission des affaires juridiques du Conseil national a procédé à l'élimination des divergences relatives à ce projet. L'objet sera traité au Conseil national lors de la session d'hiver 2019. pour protéger l'être humain et l'environnement». ${ }^{12}$ Ces propositions ne figuraient pas dans le projet du Conseil fédéral du 23 novembre 2016. Le contreprojet a également été adopté par le Conseil national le 15 juin $2018^{13}$.

6 Le texte va moins loin que celui de l'initiative populaire, notamment en matière de responsabilité. Ainsi, la responsabilité de l'entreprise ne serait engagée qu'en cas de dommages à la vie et à l'intégrité personnelles ou à la violation du droit de propriété. Les entreprises n'encourraient aucune responsabilité si elles apportaient la preuve qu'elles ont pris les mesures de protection des droits de l'homme et de l'environnement prévues par la loi pour empêcher un dommage ou qu'il leur était impossible d'influencer le comportement de l'entreprise contrôlée. Par ailleurs, la responsabilité des gestionnaires et dirigeants de la société serait exclue. ${ }^{14}$

$12 \overline{\text { Message du } 15 \text { septembre } 2017 \text { relatif à l'initiative }}$ populaire «Entreprises responsables - pour protéger l'être humain et l'environnement» (FF 2017 5999); voir généralement: Damiano Canapa/Marleen Botterbrodt/Antoine Schneebeli, Le droit Suisse des Sociétés en 2017, (2018) sui generis, 297, 301, et les références citées.

13 Le Conseil des Etats a refusé, le 12 mars 2019, le contre-projet à l'initiative pour des entreprises responsables. Le Conseil national a pour sa part maintenu sa décision d'entrer en matière le 13 juin 2019. La Commission des affaires juridiques du Conseil des Etats (CAJ-E) a ensuite décidé, le 14 août 2019, de proposer à son conseil d'entrer en matière sur le contre-projet indirect. L'objet a été retiré de l'ordre du jour de la séance du Conseil des Etats du 26 septembre 2019, afin que la CAJ-E puisse le réexaminer à la lumière de la proposition que le Conseil fédéral a annoncée le 14 août 2019, cf. BO CE 2019 à paraitre.

14 BO CN 20181054 ss. 


\section{Jurisprudence}

\section{Société simple}

\section{a) Reddition de compte: arrêt} 4A_263/2017 du 6 février 2018

\section{aa) Faits}

7 X., administrateur unique de A. SA, projette d'acquérir un parc immobilier et de regrouper des immeubles dans un fonds de type SICAV. Il sollicite un prêt hypothécaire auprès de la banque $B$. SA pour l'achat dudit parc. L'octroi de ce prêt est subordonné à la participation de la banque dans la future SICAV ainsi qu'à la signature par I., un investisseur tiers connu de la banque B. SA, d'un acte de nantissement d'un montant de CHF 10 millions (contrat-cadre). La banque informe X. que les fonds de I. sont en cours de transfert, puis refuse de verser le crédit convenu au motif que A. SA n'a pas versé $\mathrm{CHF} 10$ millions exigés à titre de sûretés selon les termes du contrat et met ensuite fin à ce dernier avec effet immédiat au motif que X. n'a pas respecté ses obligations. Une demande de reddition de compte est introduite par A. SA contre B. SA pour les correspondances entre B. SA et les notaires. La demande est rejetée en deuxième instance et $\mathrm{A}$. SA saisit le TF d'un recours en matière civile.

\section{bb)Droit}

Il s'agit de déterminer si A. SA dispose d'un droit à être renseigné sur les affaires de la banque B. SA au motif que les deux sociétés formeraient une société simple après la conclusion du contrat-cadre. Le TF ne retient pas l'existence d'une telle société. La banque s'ingère certes dans les affaires de A. SA en se réservant les rôles de directrice et de banque dépositaire de la SICAV et en prenant l'initiative de présenter un investisseur à $\mathrm{X}$. Cependant, la banque assumait essentiellement les risques d'un bailleur de fonds qui retire les bénéfices usuels de ce type d'activité (additionnés du profit supplémentaire en lien avec l'exercice de ses fonctions dans la future SICAV) ${ }^{15}$. Partant, le recours est rejeté ${ }^{16}$.

\section{b) Principe de la confiance: arrêt 4A_253/2017 du 18 juin 2018}

\section{aa) Faits}

9

C.X. possède une exploitation agricole et entretient une relation commerciale avec Z. Leurs transactions sont consignées selon un système de compte courant. En 2006, C.X. cède les biens immobiliers en relation avec son exploitation à ses deux fils, qui mettent les biens à disposition de leur père de 2007 à 2011. Suite au nonrespect du plan de remboursement du solde convenu en 2009 entre Z. et et C.X., Z. ouvre, en 2012, une action en reconnaissance de dette à l'encontre de C.X. et de ses fils en alléguant que les trois forment une société simple et qu'ils sont donc débiteurs solidaires. Le Tribunal civil de l'arrondissement de la Sarine et la Cour d'appel du Tribunal cantonal du canton de Fribourg retiennent que les membres de la famille forment une société simple. C.X. et ses fils recourent une première fois au $\mathrm{TF}$, qui renvoie la cause à la cour cantonale pour nouvelle décision. La Cour d'appel rejette une seconde fois l'appel. Les fils de C.X. interjettent un recours en matière civile.

15 Arrêt du Tribunal fédéral 4A_263/2017 du 6 février 2018, consid. 3.3.

16 Idem, consid. 4. 


\section{bb) Droit}

10

Le tiers de bonne foi ne peut se prévaloir de l'existence d'une société simple apparente que si les personnes concernées démontrent clairement, par leur comportement, qu'il en existe une entre elles. Pour apprécier cet argument, le TF précise qu'il convient de se rapporter aux règles sur la représentation de la société simple par un associé chargé de la gestion interne (art. 543 al. 3 CO) ${ }^{17}$. In concreto, $\mathrm{Z}$. pouvait croire, de bonne foi, que le solde lui était dû par une société simple composée de trois personnes: d'une part, les déclarations de $\mathrm{Z}$. indiquent qu'il considérait que C.X. et ses fils étaient tous les trois ses débiteurs. D'autre part, les comportements respectifs de C.X. et de ses fils évoquaient, de manière claire, l'existence d'une société simple $^{18}$. Le recours est rejeté ${ }^{19}$.

\section{Société anonyme}

\section{a) Droit aux renseignements et à la consultation d'un membre du CA (art. 715a CO) 20}

\section{aa) Faits}

En juin 2016, A., membre du CA de B. $\mathrm{SA}$, dépose auprès du Kantonsgerichtspräsidium du canton d'Obwald plusieurs requêtes visant à obtenir le

\footnotetext{
Idem, consid. 4.1.

Idem, consid. 4.2, 4.3.

Idem, consid. 5 .

ATF 144 III 100. A ce sujet, voir également Moschen, Corina/von der Crone, Hans Caspar, Gerichtliche Durchsetzung der Informationsrechte nach Art. 715a OR: Bundesgerichtsurteil 4A_364/2017 vom 28. Februar 2018, RSDA 2018, pp. 304-313; Sieber, Monja, Klageweise Durchsetzbarkeit von Informationsrechten des Verwaltungsrates, GesKR 2018, pp. 230-237;Naef, Monika/Rieger, Sebastian, Zur Durchesetzung von
}

droit de consulter les livres et dossiers concernant le registre des actions, le registre des ayants droits économiques et tous les procès-verbaux des séances du CA de la société. Le Kantonsgerichtspräsidium puis l'Obergericht du canton d'Obwald rejettent la demande au motif qu'il n'existe pas de base légale suffisante pour fonder une action judiciaire des administrateurs en exécution de leur droit aux renseignements et à la consultation (art. 715a CO). A. recourt au TF.

\section{bb) Droit}

12 gis: le droit aux renseignements et à la consultation des administrateurs a pour but de permettre à ces derniers de s'acquitter, de manière efficace et efficiente, de leurs tâches de direction et de surveillance. L'art. $715 a$ CO constitue donc la contrepartie de la responsabilité individuelle des membres du $\mathrm{CA}^{21}$, ce qui plaide en faveur de l'admissibilité d'une action en justice dans le cas où le droit aux renseignements et à la consultation serait refusé à l'un des administrateurs ${ }^{22}$. L'interprétation systématique conduit à la même conclusion: lorsque la loi concède un doit subjectif, ce dernier est en principe justiciable, même si le texte lé-

Informationsrechten nach Art. 715a OR, PJA 2018, pp. 600-608; Sethe, Rolf/Cetinkaya, Meltem, Entwicklungen im Gesellschaftsrecht und im Wertpapierrecht, RSJ 2018, pp. 494-500; Stoffel, Walter A., «Gute Praxis» der Unternehmensführung: was gehört sich und was funktioniert?, RSDA 2018, pp. 353-360.

21 ATF 144 III 100, consid. 5.2.2.

22 A cet égard, le TF remarque que l'action en constatation de la nullité de la décision du CA (art. $714 \mathrm{CO}$ ) n'est pas un palliatif adéquat. Une telle action - rarement admise en pratique - n'aboutirait qu'à la nullité de la décision contestée, et non à la condamnation à fournir les renseignements en question, cf. idem, consid. 5.2.2. 
gal ne l'indique pas expressément ${ }^{23}$. Dans le cas contraire, le législateur le mentionne explicitement ${ }^{24}$. Il est ainsi indifférent que la possibilité d'agir en justice ne soit pas mentionnée à l'art. 715a CO (contrairement à la situation de l'art. 697 al. 4 CO). Enfin, l'argument de la doctrine selon lequel la mise en œuvre judiciaire de l'art. $715 a$ CO permettrait, en violation des principes fondamentaux du droit de la SA, de remettre en cause les décisions du CA est écarté. Les décisions du CA sont incontestables (en dehors de l'action en nullité de l'art. $714 \mathrm{CO}$ ), car il est toujours possible d'agir directement contre la société si celle-ci, par l'intermédiaire de son organe exécutif, refuse de donner droit à une prétention ${ }^{25}$. Un administrateur qui exerce en justice ses droits issus de l'art. $715 a \mathrm{CO}$ agit donc contre la société elle-même ${ }^{26}$, sans remettre en cause les décisions du CA. Le TF admet le recours: les droits issus de l'art. $715 a$ peuvent être mis en œuvre judiciairement par une action condamnatoire 27.
23 Idem, consid. 5.2.3.1.

24 Voir par exemple l'art. 513 CO. S'agissant de l'art. 697 CO, la possibilité d'une action en justice a expressément été ajoutée à l'alinéa 4 lors de la révision du droit de la SA de 2011, dans le but de concrétiser un revirement de jurisprudence, cf. idem, consid. 5.2.3.1.

25 Idem, consid. 5.2.3.2.

26 Comme le CA ne fait qu'exprimer la volonté sociale, seule la société dispose de la légitimation passive à une telle action, cf. idem, consid. 5.2.3.2.

27 Idem, consid. 5.2.3.2. Sur le plan procédural, une telle requête doit être traitée en procédure sommaire pour des motifs de célérité et de flexibilité (l'art. 250 CPC n'est pas exhaustif), cf. idem, consid. 6.

\section{b) Actes juridiques pour propre compte dans les groupes de sociétés à caractère unipersonnel 28}

\section{aa) Faits}

13 A. Holding SA détient 100\% du capitalactions de la société A. Europe Ltd, qui détient $100 \%$ du capital-actions de la société A. 1 SA, laquelle détient 100\% du capital-actions de la société A. 2 SA. Les actions de A. Holding SA sont détenues par B. (Chief Financial Officer du groupe) et D. (Chief Executive Officer du groupe). Ces derniers sont employés par la société A. 2 SA.

14 En 2006, les contrats de travail liant B. et D. à la société A. 2 SA sont modifiés en ce sens qu'une indemnité de départ doit leur être versée si le contrat est résilié par l'employeuse avant le $1^{\text {er }}$ mars 2009. L'avenant au contrat de B. est notamment signé par D. et l'avenant du contrat de D. est notamment signé par B. Ces modifications sont approuvées à l'unanimité par l'AG de A. 2 SA, à laquelle D. - en tant qu'administrateur de cette société - et B. - en tant que représentant de la totalité des actions détenue par A. Holding SA - assistent.

Le 11 décembre 2006, A. 2 SA licencie B. La société refuse de lui payer l'indemnité de départ prévue par le contrat de travail au motif que la modification contractuelle de 2006 constituerait un contrat avec soi-même nul de plein droit.

$28 \overline{\mathrm{ATF}} 144$ III 388. A ce sujet, voir également Chenaux, Jean-Luc/Marmy, Joséphine, Opérations pour propre compte au sein de sociétés unipersonnelles: une modification bienvenue, bulletin du CEDIDAC n ${ }^{\circ} 782018$. 


\section{bb) Droit}

16

Le TF rappelle l'application de sa jurisprudence relative à l'interdiction des contrats conclus avec soi-même et de la double représentation ${ }^{29}$ lorsque la société est représentée par ses organes 30 et lorsqu'il n'y a pas d'opération pour propre compte, mais qu'il existe un conflit d'intérêts entre la société et ses organes ${ }^{31}$.

Le TF avait déjà jugé32 que lorsque l'organe contractant avec lui-même est en même temps l'actionnaire unique de la société, cette dernière n'a plus besoin d'être protégée: l'affaire correspond alors nécessairement à la volonté sociale et l'autorisation d'un organe supérieur n'est pas nécessaire 33 . In casu, le TF considère que la situation litigieuse est assimilable à celle d'un actionnaire unique, car $B$. et $D$. détiennent ensemble l'entier du capital-actions de A. Holding $S A$, qui a le contrôle exclusif des filiales et sous-

Susceptibles d'entrainer un conflit d'intérêts, ces actes sont nuls sauf si (i) l'acte a été autorisé préalablement ou ratifié ultérieurement par le représenté ou si (ii) la nature de l'affaire exclut que l'opération en cause puisse porter préjudice aux intérêts de la société, cf. ATF 144 III 388, consid. 5.1.

30 Dans un tel cas, une autorisation ou une ratification par un organe de même rang (un ou deux autres membres du CA au bénéfice d'une signature individuelle ou collective) ou par un organe supérieur (l'AG - si la partie opérant pour son propre compte est l'administratrice unique de la société) est alors requises, cf. ATF 144 III 388, consid. 5.1.

31 Dans un tel cas - contrairement aux opérations pour propre compte - le pouvoir de représentation existe en principe, mais exclut tacitement, selon la volonté présumée de la société, les actes contraires aux intérêts de cette dernière. La protection des tiers de bonne foi est réservée, $\mathrm{cf}$. idem, consid. 5.1; ATF 126 III 361, consid. 3 a.

32 ATF 126 III 361, consid. 5 (revirement de jurisprudence par rapport à l'ATF 50 II 168).

33 ATF 144 III 388, consid. 5.1; ATF 126 III 361, consid. 5 a. filiales ${ }^{34}$. La jurisprudence est donc confirmée et étendue aux opérations pour propre compte conclues au sein d'un groupe de sociétés. Le TF rejette le recours et admet que A. 2 SA était valablement représentée lors de la modification de 2006 du contrat de travail de B.35.

18 Le TF balaye par ailleurs les critiques d'une partie de la doctrine qui estimait que l'autorisation d'un organe supérieur était nécessaire, même en l'absence de conflit d'intérêt ${ }^{6}$. La révision du droit de la SA de 2008 (notamment les art. 625 et $718 b$ CO) démontre la volonté du législateur de ne pas interdire per se les contrats avec soi-même et la double représentation 37.
$34 \widehat{\text { ATF } 144 \text { III 388, consid. 5·3.1. }}$

35 Idem, consid. 5.3.2.

36 Une partie de la doctrine considérait, d'une part, que les intérêts des créanciers ne sont pas suffisamment protégés par les actions des art. $285 \mathrm{ss}$ LP et $754 \mathrm{CO}$ et, d'autre part, qu'un acte portant préjudice aux créanciers sociaux ne peut être compris dans le but de la société. De ce fait, un tel acte n'est pas compris dans le pouvoir de représentation des organes. Les créanciers devraient alors pouvoir soulever la nullité matérielle de l'acte, cf. ATF 144 III 388, consid. 5.3.2 et références citées.

37 ATF 144 III 388, consid. 5.3.2. Dans l'arrêt complet (non publié), le TF ajoute encore que les intérêts des créanciers sont suffisamment pris en compte par les dispositions sur la protection du capital social, notamment les art. 678 et 680 al. 2 CO, cf. Arrêt du Tribunal fédéral 4A_645/2017 du 22 août 2018, consid. 6 . 


\section{c) Action en responsabilité, preuve et évaluation du dommage résultant du retard dans le prononcé de la faillite 38}

\section{aa) Faits}

B. Ltd obtient la condamnation de D. SA au paiement d'un montant de $\mathrm{CHF}$ 510'521.30 plus intérêt. Dans la faillite subséquente de D. SA, B. Ltd ne reçoit qu'un acte de défaut de biens ( $\mathrm{CHF}$ 705'219.50) et se voit céder le droit de la masse d'agir en responsabilité contre les organes dirigeants de D. SA. Le 20 mai 2014, dans le cadre de l'action en responsabilité, A. (administrateur unique de D. SA) et C. SA (cabinet d'audit) - lesquels ont entre-temps démissionné de $\mathrm{D}$. SA sont condamnés à verser CHF 273'3441.90 plus intérêts à B. Ltd. Le Pretore du district de Lugano estime la valeur de ce préjudice sur la base de l'art. 42 al. $2 \mathrm{CO}$, bien que le demandeur n'ait apporté aucune preuve du montant du dommage. Ce jugement est confirmé le 9 septembre 2016 par le Tribunale d'appello du canton du Tessin. A. et C. SA déposent un recours en matière civile auprès du TF.

\section{bb) Droit}

Après avoir qualifié le dommage subi par B. de dommage indirect («par ricochet»)39, le TF précise sa jurisprudence en matière de dommage résultant du retard dans le prononcé de la faillite en raison de la violation, par les organes, de l'avis en cas de surendettement40. La

38 Arrêts du Tribunal fédéral 4A_587/2016 et 4A_597/2016 du 22 janvier 2018. A ce sujet, voir également Stoffel, Walter A./Constantin, Arnaud, Le droit des sociétés 2017/2018, RSDA 2018, pp. 287-303.

39 Arrêt du Tribunal fédéral 4A_587/2016 et 4A_597/2016 du 22 janvier 2018, consid. 3.2.

40 Idem, consid. 4.
Haute Cour confirme qu'il appartient au demandeur de faire établir un rapport d'expert pour déterminer si la perte subie par la société en faillite est au moins équivalente à celle subie par le requérant41. Dans cette constellation, si l'art. 42 al. 2 CO facilite le calcul du dommage lorsque son montant exact ne peut pas être établi, il ne décharge pas le demandeur du fardeau de la preuve ${ }^{42}$. En confirmant la décision du Pretore du district de Lugano d'évaluer le dommage sans rapport d'expert et sans bilan de liquidation, le Tribunale d'appelo a violé les art. 42 al. 2 CO et 8 CC. Le recours est admis 43 .

\section{d) Notion de juste motif au sens de l'art. $337 \mathrm{CO}$ en lien avec la no- tion d'attributions inaliénables au sens de l'art. 716a al. 1 CO44}

\section{aa) Faits}

B., président du CA de A. SA, conteste son licenciement immédiat suite à la modification qu'il a faite du logo de la société sans tenir compte des refus collégiaux déjà exprimés par son CA. A. SA soutient pour sa part que le changement de logo constitue une attribution intransmissible et inaliénable du CA et que, dans ce contexte, $\mathrm{B}$. a dès lors manqué à ses devoirs en tant qu'administrateur d'une manière suffisamment grave que pour justifier son licenciement immédiat. A. SA est déboutée en première instance. Le 29 novembre 2016, l'Obergericht du canton de Thurgovie rejette l'appel interjeté

41 Idem, consid. 4.

42 Idem, consid. 4.

43 Idem, consid. 4.1 à 4.3 .

44 Arrêt du Tribunal fédéral 4A_349/2017 du 23 janvier 2018. A ce sujet, voir également Stoffel/Constantin (n. 38). 
par A. SA, laquelle recourt en matière civile auprès du TF.

\section{bb) Droit}

Selon le TF, la décision de changer le logo de la société doit être assumée par le CA in corpore selon l'art. $716 a$ al. 1 CO (elle ne peut être déléguée sur la base de l'art. $716 b \mathrm{CO}$ ) en raison des conséquences importantes pouvant découler d'un tel changement, tant au niveau financier que pour la réputation de la société. B. aurait donc dû convoquer une séance du CA afin que celui-ci puisse collégialement se prononcer45. Le TF juge également que, dans ce contexte, la décision unilatérale de $\mathrm{B}$. de changer le logo de A. SA constitue une violation objectivement sérieuse de ses obligations en tant qu'administrateur, propre à détruire la relation de confiance existante entre lui et la société, et peut dès lors effectivement justifier un licenciement avec effet immédiat 46 . Le recours est admis 47 .

\section{e) Augmentation de capital et ménagement dans l'exercice du droit48}

\section{aa) Faits}

Z. SA dispose d'un capital-actions de 1'200 actions d'une valeur nominale de CHF 100 chacune. X. en détient 30\%, A. et $\mathrm{B}$. en détiennent ensemble $60 \%$ et les $10 \%$ restant sont détenus par la société.

45 Arrêt du Tribunal fédéral 4A_349/2017 du 23 janvier 2018, consid. 3.2-3.4.

46 Idem, consid. 4.3-4.4.

47 Idem. consid. 5 .

48 Arrêt du Tribunal fédéral 4A_531/2017 du 20 février 2018. A ce sujet, voir également Gerhard, Frank/Humbel, Claude, «Cornerstone Investors» in IPOs, GesKR 2018, pp. 257-274; Sethe/Cetinkaya (n. 20); et Stoffel/Constantin (n. 38).
En juillet 2016, A. et B. vendent leur participation au groupe U. pour CHF 16 millions.

24 Lors de l'AG de Z. SA du 18 avril 2017, le groupe U. décide d'augmenter le capitalactions de CHF 120'000 à CHF 1'620'000 (soit 15 'ooo actions supplémentaires d'une valeur nominale de CHF 100, pour un prix d'émission de CHF 100. Ce prix est sensiblement inférieur à la valeur réelle des actions, compte tenu du prix de vente des actions de A. et B. au groupe U.). Le même jour, $X$. requiert le blocage du registre du commerce à l'Office du registre du commerce du Valais central. Le 8 mai 2017, le groupe U. propose à X. de racheter ses droits de souscription préférentiels pour CHF 1'O70'268. X. refuse au motif que le montant proposé serait inférieur à la valeur réelle des actions, qui se monterait à CHF 8'249'997.60. Le $1^{\text {er }}$ juin 2017, le Juge du district de Sion rejette la requête de mesures provisionnelles. Cette décision est confirmée par le Tribunal cantonal valaisan le 4 septembre 2017. X. dépose un recours en matière civile auprès du TF.

\section{bb) Droit}

25 Le TF analyse si le principe du ménagement dans l'exercice du droit l'emporte sur le principe de la majorité. Cette question est depuis longtemps controversée en droit commercial.

26 Le TF constate que l'actionnaire minoritaire $\mathrm{X}$. avait le choix entre lever CHF 450'ooo et maintenir sa participation à $30 \%$, ou renoncer à exercer son droit de souscription préférentiel et voir sa participation diluée à $2 \%$. Les juges de Mon Repos relèvent que l'AG, pour réaliser l'augmentation projetée de $\mathrm{CHF}$ 
1'500'000, aurait également pu décider d'émettre 1'500 nouvelles actions d'une valeur nominale de CHF 1'ooo, au lieu de 15 'Ooo actions d'une valeur nominale de CHF 10o. Dans ce cas, l'augmentation de capital aurait été moins dommageable pour X., car sa participation n'aurait été diluée qu'à $13 \% 49$. L'élément décisif dans le raisonnement du TF est que les actions nouvellement émises sont destinées aux actionnaires actuels, non à de nouveaux investisseurs $5^{\circ}$. En fixant un prix d'émission nettement inférieur à la valeur intrinsèque des actions, l'AG a causé, de façon abusive et disproportionnée, un préjudice important à X.; une autre mesure moins dommageable pour ce dernier aurait permis d'atteindre le même but 51 .

Le TF rejette les arguments selon lesquels la société n'avait pas d'intention dolosive et que l'actionnaire majoritaire a proposé de racheter les droits de souscription préférentiels de l'actionnaire minoritaire. D'une part, l'application du principe du ménagement dans l'exercice du droit suppose un examen selon des critères objectifs. D'autre part, l'indemnité offerte à $\mathrm{X}$. visait exclusivement à compenser la dilution économique de sa participation, non la dilution de son droit de vote ${ }^{2}$. Le recours est admis53.

49 Arrêt du Tribunal fédéral 4A_531/2017 du 20 février 2018, consid. 3.3.

50 Dans le cas contraire, le TF aurait vraisemblablement écarté l'abus de droit, car un faible prix d'émission aurait permis d'attirer plus d'investisseurs et donc de lever davantage de fonds, cf. Arrêt du Tribunal fédéral 4A_531/2017 du 20 février 2018, consid. 3.3.1.

51 Idem, consid. 3.3.1.

52 Idem, consid. 3.3.2.

53 Idem, consid. 4. Cet arrêt s'écarte ainsi des arrêts dits «Ringier» (ATF 99 II 55 et ATF 102 II 265), dans lequel le TF faisait primer la volonté des actionnaires majoritaires sur l'intérêt des minoritaires. Fait surprenant, les juges de Mon Repos n'ont pas mentionné le revirement de jurispru-

\section{f) Requête en convocation d'une AG et abus de droit (art. 699 al. 4 CO cum art. 2 al. 2 CC) 54}

\section{aa) Faits}

28 La société Z. SA a un capital-actions de CHF 100'Ooo détenu par X. et A., à hauteur de $33 \%$ chacun, et par $\mathrm{B}$, à hauteur de $34 \%$. Du 10 février au 21 novembre 2014, B. et X. sont les administrateurs de la société, avec signature collective à deux. Lors de l'AG extraordinaire du 30 octobre 2014, X. est démis de ses fonctions d'administrateur et $\mathrm{B}$. est dès lors l'unique membre du CA. La société n'a plus d'activité depuis le $1^{\text {er }}$ janvier 2015. Le 28 novembre 2014, Z. SA dépose deux plaintes pénales contre $\mathrm{X}$. en lien, notamment, avec des infractions de gestion déloyale aggravée (art. 158 ch. 1 al. 1 et $3 \mathrm{CP}$ ) et de faux dans les titres (art. 251 ch. 1 CP) commises dans le cadre de ses activités d'administrateur.

Le 2 février 2017, X. sollicite la convocation d'une AG extraordinaire dont l'ordre du jour est la révocation de l'administrateur B. - à qui X. reproche de ne pas lui avoir fourni les comptes relatifs aux années 2014-2016 - et sa nomination à cette fonction. B. refuse la requête au motif qu'elle serait constitutive d'un abus de droit en ayant pour but de faire échec aux procédures pénales et

dence dans la présente affaire. Cela peut s'expliquer de deux façons. D'une part, l'arrêt porte sur des mesures provisionnelles; le TF s'est donc limité à un examen sous l'angle de la vraisemblance. D'autre part, les arrêts «Ringier» réservent explicitement les cas d'abus de droit. On peut également reprocher au TF de n'avoir pas proposé une règle de calcul pour la fixation du prix d'émission.

54 Arrêt du Tribunal fédéral 4A_529/2017 du 21 février 2018. A ce sujet, voir également Sethe/Cetinkaya (n. 20). 
civiles pendantes. Par demande du 24 février 2017 dirigée contre Z. SA, X. saisit le Tribunal de première instance du canton de Genève en concluant à la convocation d'une AG. Z. SA, par l'intermédiaire de B., conclut au rejet de la demande et accepte de fournir les comptes 2014-2016 si X. en fait formellement la demande. Saisi d'une demande de X., le Tribunal de première instance du canton de Genève ordonne, le 11 mai 2017, la tenue d'une AG extraordinaire avec l'ordre du jour susmentionné. La Cour de justice du canton de Genève réforme le jugement de première instance le 29 août 2017 dans le sens des conclusions de $\mathrm{Z}$. SA. X. recourt auprès du TF.

\section{bb) Droit}

3o

Le TF juge que la requête d'un actionnaire tendant à la convocation d'une AG est une conclusion pécuniaire: la valeur litigieuse se rapporte à la valeur nominale des actions souscrites (même non libérées) du requérant 55 .

31 La Haute Cour rappelle ensuite que l'exercice du droit à la convocation judiciaire d'une AG et à l'inscription d'un objet à l'ordre du jour doit respecter l'art. 2 al. 2 CC, au terme duquel l'abus manifeste d'un droit n'est pas protégé par la loi56. Elle admet que le recourant, en sollicitant la tenue d'une AG extraordinaire, ne cherchait qu'à prendre le contrôle de Z. SA dans le but de stopper les procédures pénales et civiles pendantes dirigées contre lui: $\mathrm{X}$. ne tend ainsi qu'à la sauvegarde de ses propres intérêts lesquels s'opposent à ceux de la société -

55 Arrêt du Tribunal fédéral 4A_529/2017 du 21 février 2018, consid. 1.1.2-1.1.3.

56 Idem, consid. $3 \cdot 3$ en détournant une institution juridique de sa ratio legis 57 . Le recours est rejeté58.

\section{g) Qualité d'actionnaire et communication des rapports de gestion et de révision59}

\section{aa) Faits}

32 Le $1^{\text {er }}$ octobre 2007, Z. est engagé comme managing director de A.X. SA. Son contrat de travail prévoit l'acquisition de 340 actions de la société ( $34 \%$ du capitalactions) pour un montant de $\mathrm{CHF} 34$ 'ooo. Cette dernière se réserve le droit de racheter ces actions si les rapports de travail prennent fin. En octobre 2009, A.X. $S A$ résilie le contrat de travail avec $Z$. et, en 2012, la société exerce son droit de rachat des 340 actions pour le prix de CHF 9'868. U. conteste que ce prix corresponde au prix prévu pour le rachat et dépose, en sa qualité d'actionnaire, une requête en consultation de la copie des rapports de gestion et de révision de A.X. SA (art. 696 al. 3 CO). La société ne fait pas droit à sa demande au motif que $\mathrm{Z}$. ne serait plus légitimé à exercer ses droits d'actionnaire, puisqu'il refuse illicitement de transférer les actions. Le Tribunal de première instance puis la Cour de justice du Canton de Genève ordonnent la production des documents. A.X. SA forme un recours en matière civile.

\section{bb) Droit}

33 La question litigieuse est de savoir si Z. devait être considéré comme actionnaire au moment du dépôt de sa requête en

57 Idem, consid. 4.2, 4.4 et 4.5 .

58 Idem, consid. 4.4 et 4.5 .

59 Arrêt du Tribunal fédéral 4A_248/2017 du 22 février 2018A ce sujet, voir également Stoffel/Constantin (n. 38). 
consultation de la copie des rapports de gestion et de révision. Pour le TF, le transfert de sociétariat invoqué par A.X. SA pour justifier l'absence de droits d'actionnaire de Z. n'était pas valable, car le titre d'acquisition des actions, contrairement à ce qui est exigé par l'art. 165 al. $1 \mathrm{CO}$, n'était pas accompagné d'une déclaration de cession écrite: contrairement aux affirmations de A.X. SA, le contrat de travail de $\mathrm{Z}$. ne constitue pas un acte de disposition ${ }^{60}$. Au surplus, Z. ne commet pas d'abus de droit (art. 2 al. 2 $\mathrm{CC}$ ) en refusant de céder les actions au motif que le prix de vente est 100 fois inférieur au prix convenu (exception d'inexécution, art. $82 \mathrm{CO})^{61}$. La qualité d'actionnaire de Z. ayant été reconnue, il était légitimé à faire valoir son droit à la consultation (art. 696 al. 3 CO) ${ }^{62}$. Le recours est rejeté63.

\section{h) Droit au contrôle spécial64}

\section{aa) Faits}

34 Le capital-actions de C. SA se monte à CHF 600'00o, divisé en 600 actions nominative d'une valeur nominale de CHF 1'ooo. Son CA est composé du président D., titulaire de 310 actions, et de l'administrateur E., titulaire de 30 actions. Les 260 actions restantes sont détenues par A. Sàrl et B. Le 14 juillet 2017, A. Sàrl et B. requièrent du Kantonsgericht du canton de Lucerne l'institution d'un contrôle spécial sur la base de l'art. 697b CO dans le but, d'une part, d'auditer les comptes de C. SA et de ses

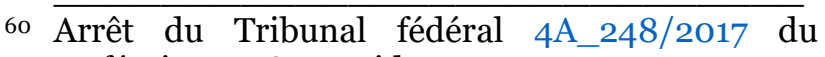
22 février 2018, consid. 3.1.

61 Idem, consid. 3.2.

62 Idem, consid. 3.2.

63 Idem, consid. 4.

64 Arrêt du Tribunal fédéral 4A_107/2018 du 29 octobre 2018. nombreuses sociétés affiliées (avec qui C. SA a conclu d'importants contrats de prêt) et, d'autre part, de déterminer la valeur réelle des actions devant être vendues par C. SA à D. et à E. Par décision du 3 janvier 2018, le Tribunal cantonal rejette la demande. A. Sàrl et B. recourent en matière civile.

\section{bb) Droit}

35 Le TF rappelle que tout actionnaire peut proposer à l'AG l'institution d'un contrôle spécial (art. 697a CO), à condition qu'il ait préalablement exercé ses droits aux renseignements et à la consultation au sens de l'art. 697 CO65. Lorsque l'AG refuse l'institution du contrôle spécial, les actionnaires peuvent requérir du juge l'institution d'un tel contrôle à condition (1) de rendre vraisemblable la violation, par les fondateurs ou les organes dirigeants, d'une disposition légale ou statuaire ayant causé un préjudice à la société ou aux actionnaires et (2) d'établir clairement le lien entre les droits des actionnaires qu'ils entendent exercer et l'objet de la requête ${ }^{66}$.

36 Les plaignants justifient leur intérêt à faire contrôler les comptes annuels de l'exercice 2015 de C. SA et de ses filiales en premier lieu par le besoin d'obtenir des informations en vue d'intenter une action en responsabilité contre $\mathrm{D}$. et $\mathrm{E}$. Ces derniers auraient manqué de diligence en accordant de nombreux prêts aux sociétés filles de C. SA. Le TF con-

65 Arrêt du Tribunal fédéral 4A_107/2018 du 29 octobre 2018, consid. 4.1 et 4.2; ATF 140 III 610, consid. 2.2; ATF 138 III 246, consid. 3.1 et 3.3; ATF 133 III 133, consid 3.2; ATF 123 III 261, consid. 3a.

66 Arrêt du Tribunal fédéral 4A_107/2018 du 29 octobre 2018, consid. 4.1 et 4.3 ; ATF 138 III 252, consid. 3.1 et 3.3 . 
firme la décision cantonale selon laquelle les requérants n'ont pas clairement indiqué en quoi l'audit spécial des comptes de 2015 aurait permis d'intenter une action en responsabilité, ce d'autant plus que l'exercice 2015 était déjà clôturé au moment de l'examen de la requête et que la décharge envers D. et E. avait été votée ${ }^{67}$. Les plaignants invoquent, en second lieu, leur intérêt à obtenir un contrôle spécial afin de déterminer la valeur réelle des actions de $\mathrm{C}$. SA devant être vendues à $\mathrm{D}$. et à $\mathrm{E}$. Le $\mathrm{TF}$ considère que les requérants n'ont pas clairement démontré (1) en quoi l'audit spécial des comptes de 2015 permettrait de déterminer la valeur réelle des actions à céder, puisque cette valeur se détermine en fonction de la situation actuelle de la société, et (2) en quoi la consultation des comptes annuels des années précédentes serait nécessaire pour déterminer la valeur actuelle des actions ${ }^{68}$. Le TF rejette le recours 69 .

\section{i) Légitimation de la qualité de titulaire d'actions au porteur en lien avec une procédure d'annulation d'un titre ${ }^{70}$}

\section{aa) Faits}

Le capital-actions de la société A. SA est composé de 20 actions au porteur. B. B.V. détient les actions $\mathrm{n}^{\circ} 11$ à 20. A. SA conteste la qualité d'actionnaire de $\mathrm{B}$. B.V. Le 30 juin 2015, une AG extraordinaire de A. SA a lieu sans que B. B.V.

67 Arrêt du Tribunal fédéral 4A_107/2018 du 29 octobre 2018, consid. 5.2 et 7.3.

68 Arrêt du Tribunal fédéral 4A_107/2018 du 29 octobre 2018, consid. 5.2 et 8 .

69 Arrêt du Tribunal fédéral 4A_107/2018 du 29 octobre 2018, consid. 10.

70 Arrêt du Tribunal fédéral 4A_501/2018 du 3 décembre 2018. n'ait été invité ni représenté. Le 19 septembre 2016, B. B.V. requiert du Zivilkreisgericht de Bâle-Campagne ouest la constatation de la nullité des décisions prises lors de l'AG extraordinaire. Parallèlement, B. B.V. dépose action en annulation des titres au porteur $\mathrm{n}^{\circ} 11-20$ (art. $972 \mathrm{CO}$ ) devant le même tribunal. Par décision du 22 août 2017, le Zivilkreisgericht annule lesdites actions (avec effet au 4 septembre 2017) et, par décision du 7 septembre 2017, constate la nullité des résolutions prise lors de l'AG extraordinaire. Ces décisions sont confirmées le 7 septembre 2017 par le Kantonsgericht de Bâle-Campagne. A. dépose un recours en matière civile.

\section{bb) Droit}

38 La question litigieuse concerne la légitimation auprès de l'AG d'un titulaire d'actions au porteur. Si une telle légitimation est en principe apportée par la possession des titres, la loi n'exclut pas la possibilité d'apporter d'autres éléments de preuve $^{71}$. En particulier, une fois le titre annulé, celui-ci est privé de sa légitimation formelle et son titulaire peut faire valoir son droit sans présenter le papier-valeur (art. 972 al. 1 CO), car il est alors placé dans la situation qui aurait été la sienne s'il avait perdu le titre ${ }^{72}$.

71 Arrêt du Tribunal fédéral 4A_501/2018 du 3 décembre 2018, consid. 3 .

72 Arrêt du Tribunal fédéral 4A_501/2018 du 3 décembre 2018, consid. 5.2. Précisons que les juges de première instance ont considéré que la procédure d'annulation permettait à B. B.V. de se légitimer auprès de la société, car la décision d'annulation a pris effet le 3 septembre 2017, soit peu avant la décision de première instance au sujet de l'action en constatation de la nullité des décisions de l'AG. 
39 L'annulation d'un titre ne préjuge pas de la situation juridique matérielle: le débiteur conserve toutes les objections qu'il pourrait soulever si le créancier possédait encore le titre, comme par exemple nier l'existence du droit ou faire valoir que le créancier n'est en réalité pas le propriétaire du titre ${ }^{3}$. Le TF considère qu'il appartient à la société de contester la qualité d'actionnaire de B. B.V., conformément à l'art. 8 CC74. A. SA n'ayant pas réussi à apporter cette preuve et reconnaissant qu'elle n'a pas invité B. B.V. à l'AG extraordinaire, les décisions prise lors de cette dernière sont nulles. Le TF rejette le recours 75 .

\section{Société à responsabilité limitée}

\section{a) Motifs d'exclusion d'un associé: ATF 144 III 394 6}

\section{aa) Faits}

40 En septembre 2014, A. Sàrl introduit auprès du Kantonsgericht du canton de Zoug une demande d'exclusion de B. Sàrl, qui détient $30 \%$ de ses parts sociales. L'action est admise au motif que la situation entre les deux sociétés est, depuis longtemps, conflictuelle. En décembre 2016, B. Sàrl fait appel contre cette décision auprès de l'Obergericht $d u$ canton de Zoug. A. Sàrl ne dépose aucune réponse dans le délai légal de l'art. 312 CPC. L'Obergericht admet l'appel et re-

73 Arrêt du Tribunal fédéral 4A_501/2018 du 3 décembre 2018, consid. 5.2.

74 Arrêt du Tribunal fédéral 4A_501/2018 du 3 décembre 2018 , consid. $3,5.1$ et 5.2.

75 Arrêt du Tribunal fédéral 4A_501/2018 du 3 décembre 2018, consid. 3 et 5.2.

76 A ce sujet, voir également Sethe/Cetinkaya (n. 20); et Wipf, Olivia/von der Crone, Hans Caspar, Ausschluss eines GmbH-Gesellschafters, RSDA 2018, pp. 569-583. jette l'action visant à l'exclusion de B. Sàrl. A. Sàrl recourt auprès du TF.

\section{bb)Droit}

41 Le TF rappelle qu'un défaut de réponse dans le délai de l'art. 312 al. 2 CPC n'a aucun impact sur la procédure d'appel77. Si une question de fait ou de droit est soulevée durant la procédure, la juridiction d'appel jouit d'une pleine cognition et ne peut considérer exclusivement les arguments d'une partie ${ }^{78}$. En l'espèce, comme la violation du devoir de fidélité par un associé est un motif justifiant son exclusion de la Sàrl - expression du caractère personnel de ce type de société 79 -, la juridiction d'appel doit se prononcer au sujet de ce motif, même si la juridiction de première instance conclut que l'état de fait n'est pas suffisamment prouvé80.

42 Afin d'apprécier l'existence d'un juste motif d'exclusion, le juge doit traiter la question du caractère raisonnable du maintien de l'associé dans la société 81 . Le TF précise que la question de l'existence d'un juste motif est tranchée en équité (art. 4 CC) par le juge, en pondérant, de manière objective, tous les intérêts en cause et en tenant compte de toutes les circonstances de l'espèce ${ }^{82}$. N'ayant considéré ni la potentielle violation du devoir de fidélité de B. Sàrl ni la situation conflictuelle entre A. Sàrl et B. Sàrl, l'Obergericht a violé le droit d'être entendu de A. Sàrl (art. 29 al. 2 Cst. et

\footnotetext{
ATF 144 III 394, consid. 4.1.1.

ATF 144 III 394, consid. 4.3.2.1.

79 Pour les références doctrinales, voir ATF 144 III 394, consid. 4.3.5.2.

80 ATF 144 III 394, consid. 4.3.5.1.

$81 \quad$ ATF 144 III 394, consid. 4.3.5.2.

82 ATF 144 III 394, consid. 4.3.6.
} 
art. $53 \mathrm{CPC})^{83}$. Le TF admet le recours et renvoie la cause à l'Obergericht ${ }^{84}$.

\section{b) Compétences du préposé au registre du commerce en cas d'opting-out ${ }^{85}$}

\section{aa) Faits}

43 20 septembre 2017, le Registre du commerce (RC) du canton de Zurich requiert du Handelsgericht du canton de Zurich de prendre les mesures nécessaires contre A. Sàrl en raison d'une carence dans son organisation (art. $731 \mathrm{bcum}$ 819 CO; art. $941 a$ al. 1 CO; art. 154 ORC). En substance, le RC fait valoir que A. Sàrl n'a pas fourni une comptabilité à jour suite à la demande de renouvellement de la déclaration d'opting-out (art. 62 al. 4 cum 83 ORC), qui avait été valablement formulée au moment de sa fondation. Le RC justifie sa demande de renouvellement au motif que, A. Sàrl faisant l'objet d'actes de défaut de biens suite à des saisies infructueuses pour dettes d'impôt et ne disposant plus d'actifs, l'Office des poursuites a demandé la radiation de la société, conformément à l'art. 155 ORC. Par décision du 10 octobre 2017, le Handelsgericht du canton de Zurich rejette la demande. Le RC dépose un recours en matière civile.

\section{bb)Droit}

44 Bien que cela ne ressorte pas de l'énoncé de l'art. $727 a \mathrm{CO}$, l'opting-out peut inter-

83 Idem.

84 ATF 144 III 394, consid. 4.4.

85 Arrêt du Tribunal fédéral 4A_589/2017 du 9 février 2018. A ce sujet, voir également Bettschart, Sébastien, L'opting-out et les attributions du registre du commerce, GesKR 2018, pp. 506-509; Sethe/Cetinkaya (n. 20); et Stoffel/Constantin (n. 38). venir lors de la fondation de la société (art. 62 al. 3 ORC) ${ }^{86}$. L'opting-out s'applique alors aux années suivantes, sauf si un associé exige un contrôle restreint (art. $727 a$ al. 4 cum 818 CO).

45 La question controversée est de savoir si le RC peut, en tout temps et sans conditions, exiger le renouvellement de la déclaration d'opting-out, conformément à l'art. 62 al. 4 ORC. Le TF retient, sur la base des art. $727 a$ al. 4 et $941 \mathrm{CO}$ et de l'art. 152 ORC, que le RC ne peut exiger le renouvellement de l'opting-out que s'il a connaissance d'un changement de circonstances laissant penser que les conditions de l'opting-out ne sont plus réunies ${ }^{87}$. Cela vaut également dans les cas où l'opting-out est intervenu au moment de la fondation ${ }^{88}$. A cet égard, la Haute Cour considère que le législateur a réglé les conditions de l'opting-out exhaustivement à l'art. $727 a \mathrm{CO}$. Ainsi, une situation financière difficile, voire un surendettement, ne sont pas de nature à remettre en cause l'opting-out et l'absence, in casu, d'organe de révision n'est pas constitutive d'une carence dans l'organisation (art. 731 b cum $819 \mathrm{CO})^{89}$. La procédure de l'art.154 ORC n'est pas destinée à provoquer la faillite de la société sans poursuite préalable dans les

$86 \overline{\text { Dans un tel cas, le préposé au registre du com- }}$ merce se contentera de la déclaration de tous les fondateurs confirmant qu'ils renoncent au contrôle restreint et de l'attestation que la société créée n'occupe pas plus de dix emplois à plein temps en moyenne annuelle, les documents visés à l'art. 62 al. 2 ORC (notamment les comptes) n'existant pas encore lors de la fondation, cf. Arrêt du Tribunal fédéral 4A_589/2017 du 9 février 2018, consid. 2.2.3.

87 Arrêt du Tribunal fédéral 4A_589/2017 du 9 février 2018, consid. 2.3.2 à 2.3.4

88 Arrêt du Tribunal fédéral 4A 589/2017 du 9 février 2018, consid. 2.3.1.

89 Arrêt du Tribunal fédéral 4A_589/2017 du 9 février 2018, consid. 2.4.2 à 2.4.3. 
cas où la société est surendettée et qu'il n'existe, par ailleurs, aucune carence dans l'organisation 90 .

\section{Société coopérative}

\section{c) Exclusion d'un associé pour justes motifs (art. $846 \mathrm{CO}$ ) 91}

\section{aa) Faits}

La SCoop Z. a pour but de gérer et d'administrer les unités locatives du site U. et de les mettre à disposition des coopérateurs exerçant des activités industrielles et/ou artisanales. X. est coopérateur de la Scoop Z. Il a été membre de sa direction (jusqu'en 2010), administrateur et directeur (tous deux jusqu'en 2012).

Lors de sa séance du 7 novembre 2012 (à laquelle X. n'a pas assisté), l'administration de la SCoop $\mathrm{Z}$. décide d'exclure $\mathrm{X}$. pour justes motifs. Il est notamment reproché à $X$. d'avoir refusé de renseigner la SCoop Z. sur les points soulevés dans un courrier du 12 octobre 2012, où il était par exemple fait reproche à Z. d'avoir adressé une facture à la SCoop Z. d'un montant de CHF 43'254 pour l'achat de 89 moteurs de store, soit CHF 486/pièce, alors que leur prix d'achat était de seulement 25 euros/pièces. A la suite du recours de X., L'AG extraordinaire du

90 Arrêt du Tribunal fédéral 4A_589/2017 du 9 février 2018 , consid. 2.4.4. Le TF considère que le $\mathrm{RC}$ a le choix entre la procédure de l'art. 154 ORC ou celle de l'art. 155 ORC si les conditions de ces deux articles sont remplies (consid. 2.4.1.). Contra Bettschart, op. cit., p. 508, qui considère qu'une société sans activité et sans actifs réalisables (art. 938a CO) doit être radiée d'office (art. 155 ORC), même dans les cas de carence organisationnelle, car une procédure de faillite ne se justifierait plus dans un tel cas.

91 Arrêt du Tribunal fédéral 4A_59/2018 du 23 août 2018.
20 février 2013 de la Scoop Z. approuve l'exclusion de ce dernier. Après avoir été débouté sur le plan cantonal, $\mathrm{X}$. dépose simultanément un recours en matière civile et un recours constitutionnel subsidiaire auprès du TF.

\section{bb)Droit}

48 Le TF constate que les juges cantonaux ont considéré l'exclusion de $\mathrm{X}$. comme justifiée: X. a acquis, pour le compte de la SCoop Z., des moteurs de stores au prix de 25 euros/pièce qu'il a ensuite facturés à la SCoop Z. pour un montant de $486 \mathrm{CHF} /$ pièce, sans qu'il ait pu démontrer un travail justifiant une plus-value de plus de $\mathrm{CHF}$ 40092. Le TF confirme que le travail de $\mathrm{X}$. ne pouvait en aucun cas légitimer une plus-value de $\mathrm{CHF} 400$, les employés de la SCoop Z. ayant euxmêmes procédé à l'adaptation des moteurs93. Ce motif conduit à lui seul la Haute Cour à conclure que le comportement de $\mathrm{X}$. est de nature à rompre le rapport de confiance entre les parties (art. 11 $\S 1$ des statuts et art. 846 al. 1 et 2 CO)94. Le $\mathrm{TF}$ rejette les deux recours et confirme l'exclusion de X.95.

\section{Doctrine}

\section{Ouvrages généraux}

49 Akeret, Fabian, Verantwortlichkeitsklagen in der Schweiz während den letzten 20 Jahren, thèse, Zurich/Saint-Gall 2018; Baker \& McKenzie (édit.), Entwicklungen im schweizerischen Wirtschaftsrecht 2017/2018, Zurich 2018;

92 Arrêt du Tribunal fédéral 4A_59/2018 du 23 août 2018, consid. 2.

93 Idem, consid. 4.

94 Idem, consid. 4.

95 Idem, consid. 5 . 
Behnisch, Urs R., Aktuelle Fragen aus dem Gesellschaftsrecht: insbesondere aus der Sicht des Notariats - Weiterbildungstagung des Verbandes bernischer Notare und des Instituts für Notariatsrecht und Notarielle Praxis an der Universität Bern vom 24./25. Oktober 2018, Berne 2018; Brändli, Beat, Verfahrensfragen im Gesellschaftsrecht, in: Dolge, Annette et al. (édits), Updates und neueste Entwicklungen im schweizerischen und internationalen Zivilprozessrecht, Zurich 2018, pp.65-82; Favre, Pascal, Transmission d'entreprise: fiscalité, régime matrimonial, succession, droit des sociétés, planification, assurances et prévoyance, retraite et préretraite, 6ème éd. actualisée et complétée, Lausanne 2018; Favrod-Coune, Pascal, Crowdfunding: analyse de droit suisse du financement participatif, thèse, Lausanne 2018; Gnos, Urs P./Hohler, Dominik/Anner, Fabienne, Gesellschaftsrecht, Entwicklungen 2017, Berne 2018; Grolimund, Pascal et al. (édits), Festschrift für Anton K. Schnyder: zum 65. Geburtstag, Zurich 2018; Jäggi, Vincent, Les actifs de la société sportive: réflexions fondamentales sur leur nature et traitement du point de vue contractuel, comptable et du droit des sociétés, thèse, Lausanne 2018; Kaempf, Markus/Härtner, Stefan, Gesellschaftsrecht (Rechtsentwicklungen EU und Kommentar), in: Wirtschaftsrecht Schweiz - EU: Überblick und Kommentar 2017/2018, Zurich/St-Gall 2018, pp. 5782; Killias, Laurent, Gesellschaftsrechtliche Streitigkeiten vor Schiedsgerichten Königsweg oder Sackgasse?, in: Grolimund, Pascal et al. (édits), Festschrift für Anton K. Schnyder: zum 65. Geburtstag, Zurich 2018, pp. 1053-1068; Kunz, Peter V./Jung, Peter/Bärtschi, Harald, Gesellschaftsrecht, 2ème éd., Zurich 2018;
Meier-Hayoz, Arthur/von der Crone, Hans Caspar/Angstmann, Luca, Wertpapierrecht, 3ème éd., Berne 2018; MeierHayoz, Arthur/Forstmoser, Peter/Sethe, Rolf, Schweizerisches Gesellschaftsrecht: mit neuem Firmen- und künftigem Handelsregisterrecht und unter Einbezug der Aktienrechtsreform, 12ème éd., Berne 2018; Merle, Philippe/Fauchon, Anne, Droit commercial: sociétés commerciales, 22ème éd., Paris 2018; Motal, Bernhard, Grundfragen des liechtensteinischen Personen- und Gesellschaftsrechts, thèse, Bâle 2018; Peter, Henry et al. (édits), Développements récents en droit commercial VI, Lausanne 2018; Raedler, David, Les enquêtes internes dans un contexte suisse et américain: instruction de l'entreprise ou Cheval de Troie de l'autorité? thèse, Lausanne 2018; Staub, Leo (édit.), Beiträge zu aktuellen Themen an der Schnittstelle zwischen Recht und Betriebswirtschaft IV, Zurich 2018; Mosimann, Michael, Corporate, contractual, and selected other legal aspects of impact investments, thèse, Zoug 2018. SA

5o Böckli, Peter/Bühler, Christoph B., Zur «Konzernverantwortungsinitiative»: rechtliche Überlegungen zu den vier Forderungen der eidgenössischen Volksinitiative «Für verantwortungsvolle Unternehmen zum Schutz von Mensch und Umwelt», Zurich 2018; Bühler, Christoph B., Konzernhaftungsrisiken und mögliche Vorkehrungen zu deren Minimierung, in: Grolimund, Pascal et al. (édits), Festschrift für Anton K. Schnyder: zum 65. Geburtstag, Zurich 2018, pp. 989-1016; von der Crone, Hans Caspar/Cahannes, Merens, Die Societas Unius Personae (SUP) aus Schweizer Sicht, in: Grolimund, Pascal et al. (édits), Festschrift für Anton K. Schnyder: zum 
65. Geburtstag, Zurich 2018, pp. 10691089; Druey, Jean Nicolas, Konzerntransparenz, in: Grolimund, Pascal et al. (édits), Festschrift für Anton K. Schnyder: zum 65. Geburtstag, Zurich 2018, pp.1017-1034; Favrod-coune, Pascal, Publication d'un prospectus d'émission dans le cadre d'une campagne de financement participatif: étude de droit comparé, in: Bahar, Rashid et al. (édits), Le droit comparé et le droit suisse, Genève 2018, pp. 223-244; Gauch, Sandro, Grundlage und Bedeutung der Business Judgment Rule in der Schweiz, thèse, $\mathrm{Zu}-$ rich 2018; Handschin, Lukas/Kenel, Luca, Voraussetzungen und Umfang der Rückerstattungspflicht gemäss Art. 678 Abs. 2 OR, in: Grolimund, Pascal et al. (édits), Festschrift für Anton K. Schnyder: zum 65. Geburtstag, Zurich 2018, pp. 10351052; Liatowitsch, Manuel, Das anwendbare Recht bei der deliktischen Haftung der Gesellschaft für ihre Organe im internationalen Konzern, in: Grolimund, Pascal et al. (édits), Festschrift für Anton K. Schnyder: zum 65. Geburtstag, Zurich 2018, pp. 225-233; Meier, Betty-Annett, Bewertung des Arbeitgebers im Internet, Zurich 2018; Müller, Roland, Die Bedeutung des Verschuldens im schweizerischen Arbeitsrecht, in: Emmenegger, Susan et al. (édits), Brücken bauen: Festschrift für Thomas Koller, Berne 2018, pp. 657-684; Müller, Roland, Arbeit im Konzern, in: Portmann, Wolfgang et al. (édits) Fachhandbuch Arbeitsrecht: Expertenwissen für die Praxis, Zurich 2018, pp. 47-83.; Oehri, Daniel, Der Sachwalter im Nachlassverfahren: ein Diener zweier Herren, Zurich 2018; Rouiller, Nicolas/Bauen, Marc/Bernet, Robert, La società anonima svizzera, Genève 2018; Schwarzmann, Simone, Die Stimmpflicht und die Offenlegungspflicht der Pensi- onskassen, thèse, Zurich 2018; Senn, Daniel, Die Beteiligung wirkungsbetroffener Dritter im Zivilprozess: dargestellt am Beispiel der Anfechtung von Generalversammlungsbeschlüssen gemäss Art. 706 f. OR, Zurich 2018; Tanner, Brigitte et al., Zürcher Kommentar, Obligationenrecht: Die Aktiengesellschaft - Generalversammlung und Verwaltungsrat, Mängel in der Organisation, Art. 698-726 und 731b OR, 3ème éd., Zurich 2018; Tawil, Joël, Les rachats d'actions propres en droit des sociétés: une approche comparée, in: Bahar, Rashid et al. (édits), Le droit comparé et le droit suisse, Genève 2018, pp. 321-338.

\section{LFus}

51 Tschäni, Rudolf (édit.) Mergers \& Acquisitions XX, Zürich 2018.

\section{Droit comptable}

52 Bucheler, Rémy, Abrégé de droit comptable: articles 957 ss CO et législation sur les sociétés et autres entités: cadres normatifs, dispositions générales, contenu des comptes annuels, comptabilité générale, comptabilité des entreprises, 2ème éd., Zurich 2018; Chardonnens, JeanPierre, Comptabilité des sociétés, 8ème éd., Hünenberg See 2018. 